\title{
Multi-agent Based Modeling Simulation about VANET
}

\author{
Wan shanshan \\ Beijing University of Civil \\ Engineering and Architecture, Beijing, \\ 100044, China \\ wss@bucea.edu.cn
}

\author{
Wang Dongliang \\ Beijing University of Civil \\ Engineering and Architecture, Beijing, \\ 100044, China \\ wangdl@bucea.edu.cn
}

\author{
Cao Qing \\ Beijing University of Civil \\ Engineering and Architecture, Beijing, \\ 100044, China \\ caoqing@bucea.edu.cn
}

\begin{abstract}
The self-organization characteristics and the interaction between a large numbers of self-organizing vehicles are complexity, to obtain a more accurate model of vehicular Ad-hoc network(VANET) and obtain a more profound comprehension of the complex behavior working mechanism of the vehicle in the VANET environment multi-agent based and bottom-up modeling approach is proposed here. It aims to describe the dynamics of VANET caused by the different behaviors of vehicular. The simulation tool for vehicular' misbehaviors is developed with multi-agent. It aims to and be able to effectively reproduce the real VANET scene. Though the multi-agent based modeling the emergent behavior and sudden existing behaviors of VANET entities are well reflected.
\end{abstract} hoc

Keywords- Multi-agent; Network Simulation; Vehicular Ad-

\section{INTRODUCTION}

The safety probelm caused by road traffic accidents has attracted more research because it has become a global public safety issues. And traffic is called the world's number one killer. Vehicular Ad hoc Network (VANET) is proposed as one of the important foundation of the intelligent transportation system for this demand [1]. VANET refers the open mobile Ad hoc network composed by the communication with each other between vehicles on the road and vehicles with fixed access points.

How to define a real vehicles movement scene and provide a macro-and micro-level accurate realistic vehicle model is one of the challenges of the VANET. Although some experience has been accumulated about the mobility model and have some mobility models been proposed, these models cannot faithfully reproduce the VANET actual application environment and more in-depth research is needed. In this paper we introduce some existing models and put forward multi-agent based model and P-AOC methodology is used. A true VANET actual application environment is reproduced by the simulation based on NetLogo platform.

\section{VANET INTRODUCTION}

VANET build a self-organizing, easy to deploy, low-cost, open structure of inter-vehicle communication network on the road. VANET can implement accident alarm, auxiliary driving, road traffic information query, passengers' communications and applications such as Internet Information Services. VANET is designed not only to improve transport efficiency but also to support reliable and secure multiple convenience for drivers passage, to make travelers more comfortable though the platform for communication between vehicles [2,3]. Though mutual exchange of state information between the vehicles, the traffic notices are notified through the VANET to the drivers in advance. And drivers are recommended to make timely and appropriate driving behavior decision under the circumstances. This will effectively enhance the driver's attention, to improve the safety of driving.

Vehicles can be positioned using satellite communication system. The vehicles within a certain range can fulfill automatically interconnected communication through a multi-hop manner. Each vehicle is not only the transceiver but also the router. The information transmission of the individual vehicle speed, acceleration, position, and other information can guarantee the normal operation of the vehicles and traffic warning. And vehicle can communicate with roadside facilities through the base station in order to get more services.

The key problem for VANET to be solved is to study the behavior of the drivers and the vehicles. That focuses on the problem of the relationship between the individual vehicles and other vehicles. Through the study of the interaction between the large numbers of vehicles, a more profound understanding of the different features of the vehicle from microscopic model of vehicle movement dynamics is obtained. Only with the solution the problem, the scene of the car VANET can be faithfully reproduced and NetLogo/HubNet platform mobile traces for ns-2 network simulation platform is used for protocol performance assessment. The main problems for VANET model is shown as followings.

Traffic Management Problem. Traffic Management means the guide, organization and constraint to the vehicles and passengers on the road. It includes how to separate traffic, how to allocate traffic flow, how to divide the lanes as well as a variety of vehicles priority. To study the traffic management is to depict the structure of the traffic system, and obtain a more profound understanding of the dynamics of vehicle mobility model from the macro. It includes: To study the initial position distribution of the different kinds of vehicles in the simulation region. To study the traffic density 
distribution in the simulation time. To study the number of lanes, road priority and the length of the road and real-time dynamic traffic assignment. To study the vehicle Interaction distance and the influence for traffic flows caused by acceleration or brake.

Driver and vehicle behavior problem. It mainly discuses the inter-relationship between the individual vehicles and their vehicles and obtains a more profound understanding of the dynamics of vehicle mobility model from the macro. It includes: To study the influence on mobility model caused by driver's personalized character. To study how the driver's behavior guide the mobility model. To study how to obtain and improve their reaction behavior based on the local and sharing function.

Vehicular mobility constrained problem. In order to reproduce the vehicle mobile mode more faithfully, vehicle movement constraints must be studied which includes each vehicle's static and dynamic constraints. It includes: To study the space layout about the traffic occurred sources, attract distribution hub and road density. To study the geographic constraint problem that must be dealt with such as highways, streets, intersections and traffic lights and

\section{VANET P-AOC MODELING}

\section{A. The existing models introduction}

Literature studies have shown that the results of the performance of the VANET greatly depend on the mobility model $[4,5]$. Several models have been proposed to generate nodes movement mode where mobility is considered in the wireless network simulation. The most famous models such as the random waypoint model, random walk model, the reference point group mobility model, node catch-up model, and Gauss-Markov model have involved in the randomly generated topology boundary linear, fixed speed mobile [6,7].

Further researches increase pause time, the boundary reaction, acceleration or deceleration of the node and so on. Simplification particularly led to the success of the random waypoint model. But composed to the real-world behavior some non-realistic mobile mode might occur for the internal features of this mobility model. The new open source tools can be used to produce a vehicle mobility model. Most of them can produce traces for network emulator. Some models such as random mobility model and Manhattan model merely model the basic movement constraints and almost there is no micro movement. GEMM [8] is an extended model of BonnMotion, but it limited to a simple attraction between RWM modeling.

\section{B. Vehicular mobility modeling based on P-Aoc}

Unlike traditional Multi-Agent system the main characteristics of the AOC model is to highlight the smallest and easiest members of a complex system. The complex system is based on the following behavioral characteristics of entities which are autonomy, emergence, distribution, adaptability and self-organization. In the AOC environment complex system entities are defined and these entities can perform some initial behavior and three complex behaviors which are sudden behavior, purposeful behavior, and sudden purpose behavior. Independent of the AOC environment defines entity independent, synthesis independent, emergent autonomy of independent and computing systems.

The limitations of the of traditional AOC modeling method considered the AOC modeling based on participants methods is introduced for the AOC model, That's, Participator AOC(P-AOC)modeling method. The so-called participant modeling methodology for analysis, modeling and simulation of complex systems is characterized by a participant cooperation, which improves problem-solving ability of the system to the real participants. This method adds a new dimension to the AOC model. AOC model can be run not only according to the rules, but also through the direct participation of the people. During the simulation of the participants, the participants play the behavior of a system and it can control part of the system. P-AOC modeling method increase interaction to the AOC modeling method. In addition, NetLogo/HubNet platform provides a convenient means of simulation for the P-AOC modeling.

\section{SIMULATION EXPERIMENTS}

\section{A. Experimental program}

Around the dynamic characteristic of VANET a prototype of P-AOC method is used here and the simulation is based on computing platform NetLogo/HubNet.

Prototype P-AOC method is taken as the main line to start the study. It Includes two processes, that's, the artificial entity AOC process and participants P-AOC process. Specific technical route is shown in figure 1.

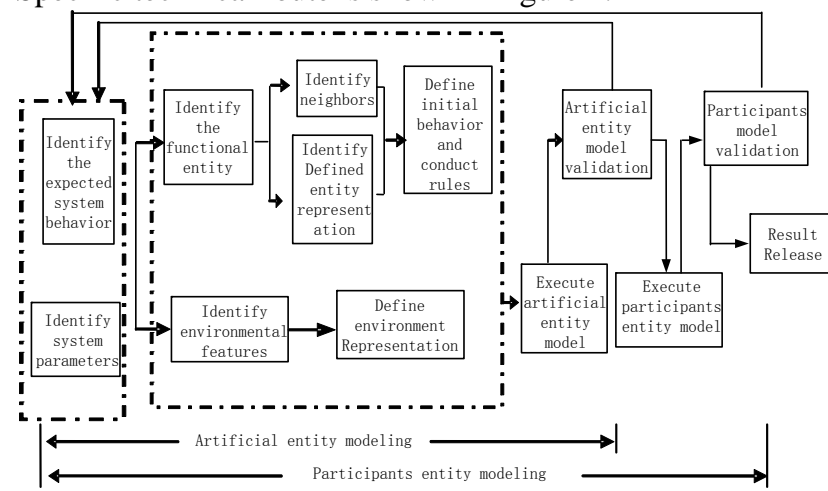

Figure 1. Technology route

1) Artificial entity AOC process.

Firstly, a multi-autonomous entity computational model is built based on existing mobility models. Next, take the hypothetical model into an implementation prototype. Then adjust the prototype if did not produce the desired effect.

2) P-AOC process.

After the artificial entity AOC model is verified the participants AOC process is executed. Because compared to those rules that are written by the scenes writer human behavior is often more variable. If the expected effect did not produce the prototype is adjusted and the participants of the AOC process will happen again. 
The above process is a process of repeated cycles. And the target is to obtain an ideal model so that it can reflect the dynamics of the network self-organization.

\section{B. NetLogo Based VANET modeling}

VANET is a distributed, non-center, high dynamic and changing network. Its environment is critical for the entire VANET running and state change. The activities of the vehicle MANET can be simplified as the interactions of the environment and the body, according to the above description of the structure and rules of the environment and the body, the following environment and the body parameters and variables are set.

Road quantity, it means the road which has no turning change to sections. Intersection quantity, it means the crossing equipped with a traffic light. Road priority, it means sections which need to be dredged firstly when road traffic congestion occurs. Emergencies, it means road experience events such as traffic control, temporary barricades.

Vehicles quantity. It means the number of vehicles in the road traffic and the value is dynamic. Driving distance, it refers to the average driving distance from source target location to destination location and cars exit the selfcontained network after the travel distance is completed. Congestion index, it is set in accordance with congestion value obtained by the traffic reports statistics, and its value is dynamic. Vehicle following proportion which refers to the proportion of the vehicle that driver likes to follow the front vehicle.

Manual adjustment capability. The main factor of the PAoc based modeling is the system for regulating control by manual including the behavior of the environment settings and the entity correction.

\section{Model Implementation and Analysis}

NetLogo platform used in the experiment is multi-agent software tool which is developed by the American Northwestern University's Center for Connected Learning. It has a good graphical interface and programming interface, users can easily adopt the method of multi-agent simulation to simulate complex adaptive systems. Its core is an objectoriented framework, and the user can define the system in a variety of subject and the subject's properties and methods. The software is applicable to a variety of complex systems model created, including sociology, economics, information science, and art. Here utilization NetLogo platform is applied to achieve VANET model.

In the model, road quantity is between 50 and 100 and the roads include one-way and two-way which are shown by the arrow. Intersection quantity is between 10 and100, intersection is indicated by a solid dot. The congested intersection is indicated with a large solid dot. The greater dot means more serious congestion serious. Road priorities and emergencies are setting with switches. The number of vehicles is between 100 and 2000, the thickness of the road constituting line represents the state of roads and the thicker line indicates more serious status.

The vehicle traveling distance is set to a value between the longest distance and the shortest distance of all roads.
Congestion index is set to a value in the dynamic between 0 to10 according to the statistics of road traffic conditions. Vehicle following proportion is set to a value between 1 and 100. The participant switch is set to adjust the prevailing conditions of the road rules and vehicle body attributes, selforganization behavior of the vehicle can be better observed.

Vehicle distance network is a complex and volatile dynamic behavior. The experiment runs continuously for 24 hours and the test data is recorded. Figure 2 shows the experimental simulation interface, the entity attributes setting buttons and control switches buttons are on the left. The right is simulation environment and the selforganization characters of VANET can be shown clearly.

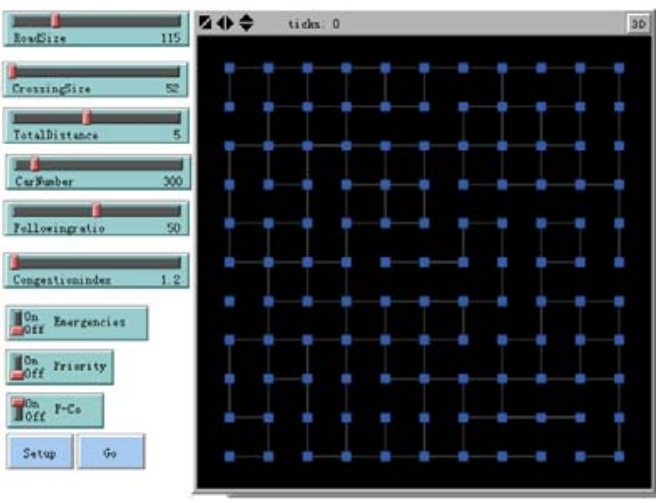

Figure 2. Experimental simulation interface

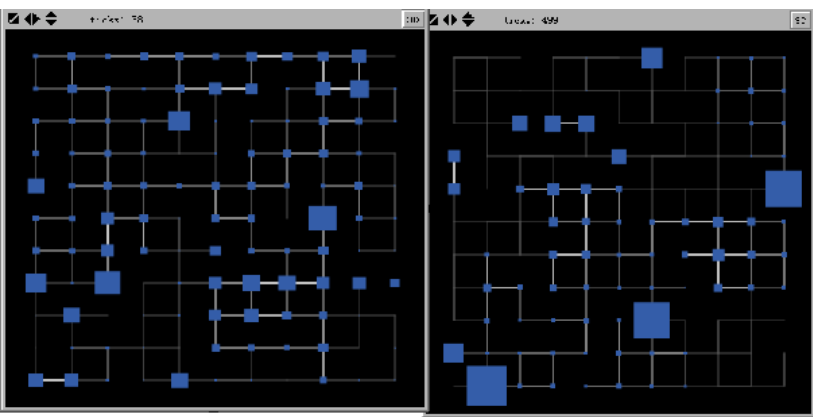

Figure 3. Artificial entity AOC model
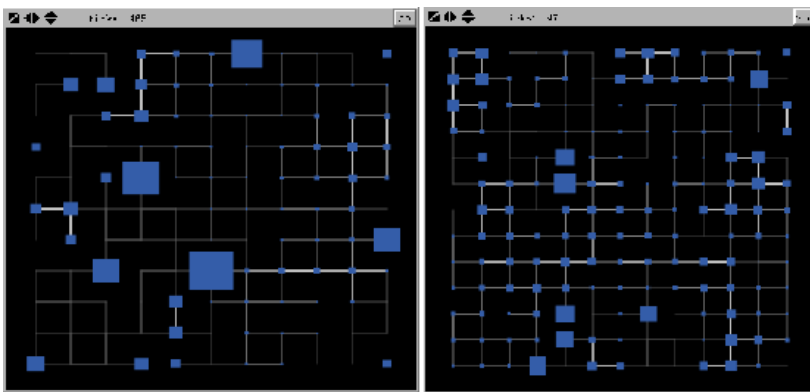

Figure 4. P-AOC model

Figure 3 pickups 3 typical times 8:00 and 10:00 and artificial control is not involved in this case. It can be seen that a vehicle entity has emergent behavior and sudden 
existing behaviors and more concentrated places appear suddenly and the congestion cannot eliminate within a short period of time.

Figure 4 is the status after the artificial intervention is start. The self-networking behavior of VANET gradually transformed into stable behavior patterns. The interaction behavior of the entities is well represented. The figures also show that the model simulates real vehicle self-organizing behavior accurately and provide a good platform for the next step of study.

\section{CONCLUSIONS}

Multi agent based P-Aoc VANET modeling method is proposed here. It aims to and be able to effectively reproduce the real VANET scene. Though the multi-agent based modeling the emergent behavior and sudden existing behaviors of VANET entities are well reflected. Analysis of the key factors which affect VANET are analyzed, the attributes and parameters of environment and vehicle entity are extracted and setup. Though NetLogo experimental platform vehicles self-organized characteristics and inter influence of a large number of self-organization vehicles are researched. The experiments show that the method with the use of the complex system theory and simulation technology faithfully reproduces the real scene of the VANET and provides a starting point for further research in the problem.

\section{ACKNOWLEDGMENT}

The authors wish to acknowledge the support of Beijing Excellent Young Talent Training Plan (No. PHR201108206).

\section{REFERENCES}

[1] B. FIEBIG.European traffic accidents and purposed solutions [A].Proc of the ITU-T Workshop on Standardization in Telecommunication for Motor Vehicles[C]. 2003. 24-25.

[2] C. PASSMANN, C. BRENZEL,R. MESCHENMOSER.Wireless vehicle to vehicle warning system[A].SAE2000 World Congress, Detroit, MI[C]. USA, 2002.

[3] C.Y. Chang, Y. Xiang, M.L. Shi. Development and status of vehicular ad hoc network. Journal on Communications.Vol.28 No.11. 2007.

[4] J. Yoon, M. Liu, and B. Noble, Random waypoint considered harmful. in INFOCOM, 2003.

[5] D. R. Choffnes and F. ann E. Bustamante, An integrated mobility and traffic model for vehicular wireless networks, in VANET '05: Proceedings of the 2nd ACM international workshop on Vehicular ad hoc networks. New York, NY, USA: ACM Press, 2005, pp. 69-78.

[6] C. Bettstetter. Mobility modeling in wireless networks:Categorization, smooth movement, and boder effects. In ACM SIGMOBILE'01, volume 5, pages 55-67, 2001.

[7] B. Wang, B. Y. Yan, D. Yuan . The basic study of the features of the ad hoc nodes mobility model. Journal of Sichuan University. 2006, 42(1):68-72.

[8] M.J. Feeley, N.C. Hutchinson, S. Ray, Realistic mobility for mobile ad hoc network simulation, in Lecture Notes in Computer Science (LNCS), Vol. 3158, pp. 324-329, 2004. 